\title{
L'incidence des anomalies chromosomiques de structure sur la spermatogenèse chez l'homme
}

\author{
JM Luciani, MR Guichaoua \\ Laboratoire de Cytogénétique et d'Embryologie, Faculté de Médecine, \\ bd de Pierre-Dramard, 13326 Marseille Cedex 15, France
}

(28® Réunion de la Société Française pour l'Étude de la Fertilité; Paris, 19-21 octobre 1989)

\begin{abstract}
Résumé - Les remaniements de structure des autosomes s'accompagnent de façon significative d'un trouble de la spermatogenèse. Les études méiotiques récemment pratiquées, sur des spermatocytes humains au stade pachytène, chez des hommes infertiles porteurs hétérozygotes de tels remaniements, ont constamment montré plusieurs éléments: un asynapsis au niveau des points de cassure, une association de la figure de translocation avec les chromosomes sexuels et la fréquence de la présence des chromosomes acrocentriques. Deux hypothèses sont essentiellement avancées pour tenter d'expliquer l'effet stérilisant des anomalies de structure des autosomes : l'arrêt de la spermatogenèse résulte : 1) d'une étroite interaction entre le chromosome $X$ et les segments remaniés des autosomes; 2) d'un défaut d'appariement des segments chromosomiques au voisinage des points de cassure.
\end{abstract}

anomalies chromosomiques / méiose / infertilité / homme

Summary - Consequences of structural chromosomal abnormalitles on human spermatogenesis. Infertility due to gametogenic failure is frequently associated with structural autosomal abnormalities. Recent meiotic studies at the pachytene stage undertaken in human infertile heterozygous carriers for such rearrangements have regularly shown a synaptic failure around the breakpoints, an association of the translocation figure with the sex chromosomes and the frequent involvement of the acrocentric chromosomes. Two main models were proposed to explain the male sterilizing effect of autosomal rearrangements: the impairment of spermatogenesis could be the result of: 1) the XY-autosome interaction; 2) the pairing disruption around the breakpoints at the pachytene stage. They could contribute significantly to germ-cell atresia.

chromosomal abnormalities / meiosis / infertility / man

La suspicion de l'existence d'anomalies chromosomiques au cours des stérilités masculines date de 1957 lorsque Ferguson-Smith et al, étudiant la chromatine sexuelle de 91 sujets présentant soit une azoospermie, soit une oligospermie sévère, découvrirent un corps de Barr chez 10 d'entre eux. Un lien, jamais démenti, était établi entre les anomalies chromosomiques et la stérilité; il trouva sa confirma- tion 2 ans plus tard avec la démonstration par Jacobs et Strong (1959) de la constitution chromosomique $47, X X Y$ de ces sujets, atteints du syndrome de Klinefelter.

Depuis ces découvertes initiales, les différentes investigations cytogénétiques pratiquées dans les stérilités masculines ont montré que non seulement les anomalies des chromosomes sexuels mais aussi les anomalies affectant les autosomes pou- 
vaient perturber le processus de la gamétogenèse.

Actuellement, à partir des données globales des études les plus importantes, totalisant plus de 11000 sujets (Kjessler, 1972; Koulischer et Schoysman, 1975; Chandley, 1979; Zuffardi et Tiepolo, 1982; Retief et al, 1984; Anès, 1985; Dutrillaux, 1985; Bourouillou et al, 1987), l'incidence des anomalies chromosomiques sur l'infertilité peut être estimée à $5,91 \%$ dont $4,44 \%$ impliquant les chromosomes sexuels et $1,46 \%$ concernant les autosomes (tableau I). Cette fréquence d'anomalies chromosomiques est très significativement supérieure à celle constatée dans la population générale (Hook et Hamerton, 1977).

Les aberrations des chromosomes sexuels sont les plus fréquemment observées (tableau I). Parmi celles-ci, la constitution chromosomique 47, XXY est très largement prédominante puisqu'elle représente plus de $80 \%$ des anomalies. Quel- ques anomalies de structure du chromosome $Y$ sont également observées. Si leur fréquence absolue est faible, par contre leur intérêt dans l'étude de la cartographie génique du chromosome $Y$ a été considérable, en particulier dans la mise en évidence de facteurs influençant la spermatogénèse, situées sur le bras long.près de la zone hétérochromatique fluorescente, à la jonction des bandes q11 et q22 (DevictorVuillet et al, 1971; Tiepolo et Zuffardi, 1976; Hartung et al, 1988).

Bien que beaucoup plus rares $(1,46 \%$, tableau (), les anomalies de structure des autosomes ont un impact certain sur la spermatogenèse. Les translocations robertsoniennes sont 10 fois plus fréquentes chez les hommes infertiles que chez les nouveau-nés $(7,1$ contre $0,6 \%$ ) . Les translocations réciproques le sont 4 fois plus $(4,2$ contre $0,8 \%)$. Une étude collaborative des cytogénéticiens français (1986) a montré que le risque d'infertilité est 2 fois plus élevé chez les sujets porteurs d'inver-

Tableau I. Nombre, pourcentages et types des diverses anomalies chromosomiques observées dans les stérilités masculines.

\begin{tabular}{|c|c|c|c|c|c|}
\hline $\begin{array}{l}\text { Nombre } \\
\text { de sujets } \\
\text { examinés }\end{array}$ & $\begin{array}{c}\text { Nombre } \\
\text { d'anomalies } \\
\text { chromosomiques }\end{array}$ & $\begin{array}{r}\text { Anom } \\
\text { chromosc } \\
\text { (nombre }\end{array}$ & $\begin{array}{l}\text { alies des } \\
\text { omes sexuels } \\
\text { et types) }\end{array}$ & $\begin{array}{r}\text { Anomal) } \\
\text { autosomiq } \\
\text { (nombre et t }\end{array}$ & ies \\
\hline \multirow[t]{2}{*}{11057} & \multirow[t]{2}{*}{$654(5,91 \%)$} & \multicolumn{2}{|c|}{$492(4,44 \%)$} & \multicolumn{2}{|l|}{$162(1,46 \%)$} \\
\hline & & $\begin{array}{l}X X Y \\
X Y Y \\
X X Y Y \\
X O / X Y \\
X X \\
\operatorname{Inv} Y \\
\text { Del } Y \\
Y_{R} \\
t(X \text { ou } Y)\end{array}$ & $\begin{array}{r}393(3,55 \%) \\
23(0,20 \%) \\
3(0,02 \%) \\
19(0,17 \%) \\
15(0,13 \%) \\
20(0,18 \%) \\
10(0,09 \%) \\
1(0,009 \%) \\
2(0,018)\end{array}$ & $\begin{array}{l}\text { translocations } \\
\text { robertsoniennes } \\
\text { réciproques } \\
\text { inversions } \\
\text { autres }\end{array}$ & $\begin{array}{l}79(0,71 \%) \\
47(0,42 \%) \\
\\
11(0,10 \%) \\
28(0,25 \%)\end{array}$ \\
\hline
\end{tabular}

Kjessler, 1974; Koulischer et Shoysman, 1975; Chandley, 1979; Zuffardi et Tiepolo, 1981; Retief et al, 1984; Anès, 1985; Dutrillaux, 1985; Bourouillou et al, 1987. 
sions péricentriques que dans la population générale. La diminution de la fertilité paraît plus prononcée pour les grands chromosomes que pour les petits, et il est possible que les inversions péricentriques du chromosome 1 soient particulièrement associées à une stérilité masculine. La cause de la stérilité est une défaillance de la gamétogenèse affectant généralement les dernières étapes de la méiose et la maturation des spermatides, et conduisant à une oligospermie sévère, parfois à une azoospermie.

Les études méiotiques récemment pratiquées, sur des spermatocytes au stade pachytène, chez des hommes infertiles porteurs hétérozygotes de remaniements purement autosomiques (Luciani et al, 1984; Rosenmann et al, 1985; Saadallah et Hulten, 1985; Chandley et al, 1986; Gabriel-Robez et al, 1986a, b; Batanian et Hulten, 1987; Johannisson et al, 1987) permettent d'avancer deux principales hypothèses pour tenter d'expliquer l'effet stérilisant de tels remaniements : l'arrêt de la spermatogenèse résulte : 1) d'une étroite interaction entre le chromosome $X$ et les segments remaniés des autosomes; 2) d'un défaut d'appariement (asynapsis) des segments chromosomiques remaniés au voisinage des points de cassure.

\section{L'INTERACTION X-AUTOSOME}

C'est au cours d'un travail tentant d'expliquer l'arrêt de la spermatogenèse chez des drosophiles mâles stériles, porteuses de translocations $X$-autosomes, que Lifschytz et Lindsley (1972) ont postulé que l'inactivation du chromosome $X$ représente un mécanisme indispensable au déroulement normal de la spermatogenèse, et que tout facteur interférant avec cette étape essentielle de la prophase méiotique est susceptible de perturber le métabolisme bio- chimique de la cellule germinale en voie de différenciation et de provoquer l'arrêt de son développement. Cette hypothèse a été étendue par Forejt $(1974,1979,1982)$, Forejt et Gregorova (1977), Forejt et al (1981), à la suite de travaux effectués chez la souris, aux remaniements purement autosomiques : translocations robertsoniennes et réciproques. Ces auteurs ont trouvé une association méiotique spécifique entre la figure de translocation et le chromosome $X$ : ce contact altérerait l'inactivation normale du chromosome $X$ et conduirait au blocage de la spermatogenèse.

Chez l'homme, le lien entre l'association $X Y$-autosome (fig 1) et la défaillance de la spermatogenèse a récemment été démontré à partir d'analyses du stade pachytène chez des sujets porteurs à l'état hétérozygote de translocations robertsoniennes 13q;14q (Luciani et al, 1984; Johannisson et al, 1987), 14q;21q (Rosenmann et al, 1985 ) et de translocations réciproques (Chandley et al, 1986, Gabriel-Robez et al, 1986b; Luciani et al, 1987; Johannisson et al, 1987). Un taux élevé d'association XYautosome, variant de 20 à $93 \%$ a toujours été observé, les taux les plus élevés étant notés lorsqu'un chromosome acrocentrique est impliqué dans la translocation (tableau II). Par ailleurs, l'association intéresse toujours la région des points de cassure où un défaut d'appariement est également constant (fig 2). Les segments autosomiques non appariés peuvent alors s'associer au segment différentiel non apparié du chromosome $X$. Cette association pourraît être responsable de la réactivation transcriptionnelle du chromosome $X$ pendant le stade pachytène de la première division méiotique chez l'homme (de Boer et Branje, 1979; Davisson et al, 1981; Forejt, 1982; Johannisson et al, 1983, 1987; Luciani et al, 1984, 1987; Rosenmann et al, 1985). A l'appui de cette hypothèse vien- 


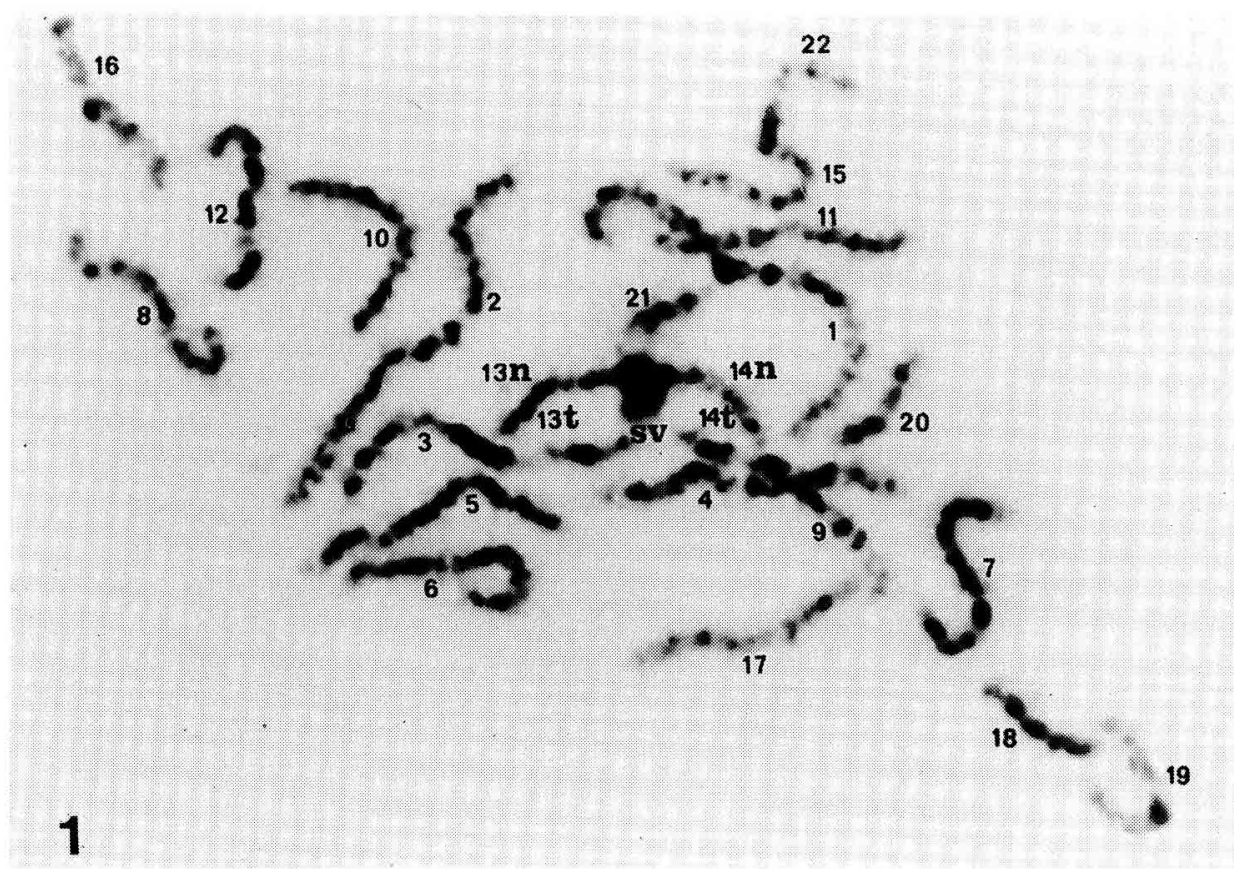

Fig 1. Translocation robertsonienne 13q;14q. Noyau de spermatocyte humain au stade pachytène dans lequel tous les bivalents autosomiques et le trivalent 13-14 ont été identifiés. Noter l'association du trivalent avec la vésicule sexuelle (sv) $(13 n, 14 n$ : chromosomes normaux; 13t,14t: chromosome transloqué).

nent s'inscrire les observations de Hotta et Chandley (1981) chez la souris où les enzymes liées au chromosome $X$ sont significativement plus élevées chez les sujets stériles que chez les témoins.

A l'inverse, Viguié et al (1982) puis Ratomponirina et al (1985) et Gabriel-Robez et al (1986b) ont postulé que l'inactivation naturelle du chromosome $X$ s'étend aux segments autosomiques asynapsés adjacents. Cette hypothèse est basée sur l'observation d'excroissances latérales apparaissant le long du segment autosomique asynapsé. Cet aspect est caractéristique de la condensation des segments non appariés des chromosomes $X$ et $Y$ au cours du stade pachytène précoce. De tels chan- gements s'accompagnant d'images d'arrêt de la transcription des gènes ribosomiques ont été observés par Guichaoua et al chez la souris (en préparation). Réactivation du chromosome $X$ ou activation de segments autosomiques mettent l'accent sur l'intégrité nécessaire des relations chromosomes sexuels-autosomes au cours de la méiose. Celles-ci sont obligatoirement perturbées au cours des translocations $X$ autosomes (pour revue, voir Quack et al, 1988 ) et $Y$-autosomes (Gonzalez et al, 1981; Laurent et al, 1982). Ces anomalies sont responsables de stérilité par arrêt de la spermatogenèse; celui-ci est d'autant plus précoce que l'altération des relations $X Y$-autosome est plus importante. 


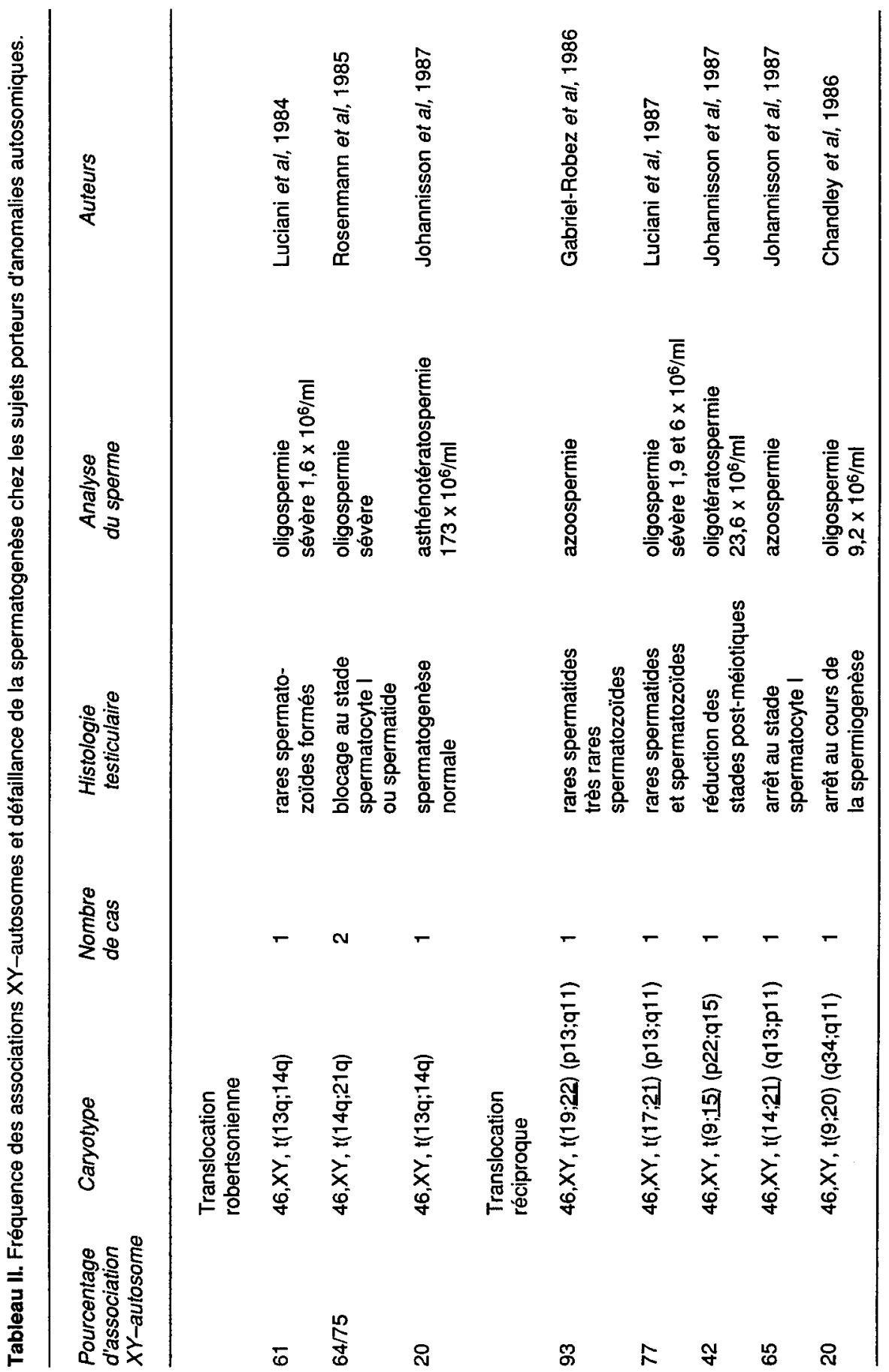




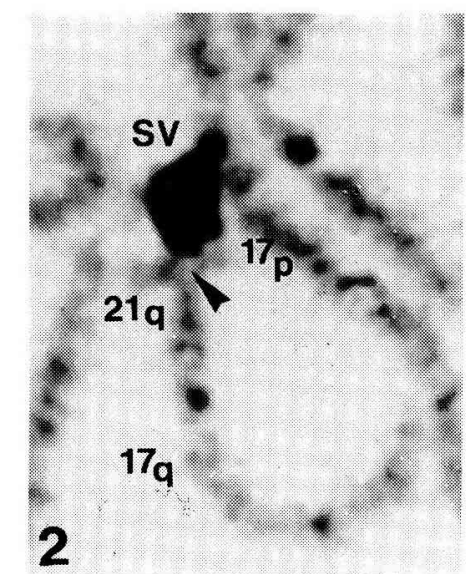

Fig 2. Translocation réciproque 17q;21q. Détail du quadrivalent 17-21 associé par les bras courts des chromosomes 21 avec la vésicule sexuelle (sv). Noter l'asynapsis (flech $\theta$ ) au niveau des points de cassure (p: bras court; q: bras long).

\section{LES ANOMALIES DE L'APPARIEMENT}

L'intéraction $X Y$-autosome n'est probablement pas l'unique cause de défaillance de la spermatogenèse.

En 1974, Miklos attirait l'attention sur l'importance des appariements des chromosomes au cours de la méiose pour assurer le développement d'une fertilité normale. La réduction ou l'absence d'appariement pourrait conduire à une altération de la spermatogenèse. Proposée tout d'abord pour les chromosomes sexuels, cette hypothèse a été étendue par Burgoyne et Baker (1984) à l'ensemble des anomalies chromosomiques de nombre et de structure, car toutes ont un point commun : la présence de régions asynapsées. Ainsi, certaines stérilités découvertes chez des sujets porteurs de remaniement structuraux et pour lesquels aucune association $X Y$-autosome n'a été observée pourraient relever de ce processus : translocations complexes (Saadallah et Hulten, 1985, $t(2,4,9)$; Johannisson et al, 1988, $t(9,12,13))$ et inversions du chromosome 1 (Gabriel-Robez et al, 1986a; Guichaoua et al, 1986; Batanian et Hulten, 1987; Chandley et al, 1987).

Ce modèle a l'avantage de pouvoir être appliqué à la spermatogénèse et à l'ovogenèse, tandis que l'effet stérilisant $X Y-$ autosome est limité au sexe mâle. Or, d'après certaines observations effectuées chez la souris, les femelles porteuses d'anomalies, ayant chez le mâle un effet stérilisant, ont une réduction du nombre d'ovocytes, de la taille des ovaires et de la durée de leur vie génitale (Mittwoch et al, 1984; Burgoyne et al, 1985). Dans l'espèce humaine, une ménopause précoce a été rapportée chez deux sœurs porteuses d'une inversion péricentrique du chromosome $X$ (Soler et al, 1981). De même, Speed (1988) attribue à de nombreuses anomalies d'appariement le fort taux d'atrésie qui caractérise le développement de l'ovocyte fœtal humain.

Chacun des deux modèles proposés pour expliquer la défaillance de la spermatogenèse chez les sujets porteurs d'aberrations de structure des chromosomes, l'association XY-autosome et des anomalies de l'appariement, est suffisant en luimême, mais ils peuvent aussi se conjuguer pour aggraver les altérations du processus.

\section{LE RÔLE DES CHROMOSOMES ACROCENTRIQUES}

La présence d'un chromosome acrocentrique semble intervenir pour favoriser l'association de la figure de translocation avec la vésicule sexuelle. Au cours de la méiose normale, les chromosomes acrocentriques sont naturellement associés à la vésicule 
sexuelle. Le taux moyen d'association pour chaque chromosome acrocentrique est de $3,4 \%$ (Guichaoua et al, en préparation). La présence de segments asynapsés peut être à elle seule responsable de l'association $X Y$-autosome par un phénomène d'attraction entre les segments asynapsés de la figure de translocation et le segment différentiel du chromosome $X$ dans la vésicule sexuelle. Cet asynapsis serait responsable des taux d'association relativement bas observés dans les translocations ne faisant intervenir aucun chromosome acrocentrique.

L'implication d'un ou de deux chromosomes acrocentriques dans la translocation favoriserait le contact avec la vésicule sexuelle. Ainsi, les deux événements, asynapsis et présence de chromosomes acrocentriques, agiraient en synergie, expliquant le fort taux d'association avec la vésicule sexuelle (tabieau II).

\section{FRÉQUENCE D'ASSOCIATION XY- AUTOSOME ET DEGRÉ D'ATTEINTE DE LA SPERMATOGENĖSE}

Les diverses investigations cytogénétiques pratiquées chez les hommes infertiles (vide supra et Marmor et al, 1980) ont montré que la fréquence des anomalies chromosomiques augmente notablement lorsque le nombre de spermatozoïdes est inférieur à 10 millions $/ \mathrm{ml}$. Les valeurs les plus basses du spermogramme sont observées chez les sujets porteurs de translocations robertsoniennes et de translocations réciproques comportant un chromosome acrocentrique. Les études méiotiques ont, par ailleurs, montré une fréquence élevée de l'association $X Y$ autosome quand un ou deux chromosomes acrocentriques sont impliqués dans la translocation (tableau II). Ainsi, une relation positive semble exister entre taux d'as- sociation $\mathrm{XY}$-autosome et atteinte de la spermatogenèse.

En conclusion, bien que la cause de la défaillance de la spermatogenése chez les sujets porteurs de remaniements chromosomiques de structure à l'état hétérozygote soit toujours discutée, plusieurs éléments susceptibles de l'expliquer sont constamment retrouvés : 1) l'asynapsis au niveau des points de cassure; 2) l'association de la figure de translocation avec les chromosomes sexuels; 3) la fréquence de la présence des chromosomes acrocentriques dans ces remaniements stérilisants; 4) la prévalence des translocations impliquant un ou deux chromosomes acrocentriques chez les sujets ayant une atteinte sévère de la spermatogenése (moins de 10 millions $/ \mathrm{ml}$ ) et un fort taux d'association $\mathrm{XY}$-autosome.

\section{RÉFÉRENCES}

Anès A (1985) Chromosomes et troubles de la spermatogenèse. Thèse de doctorat en Médecine, Marseille

Batanian J, Hulten MA (1987) Electron microscopic investigations of synaptonemal complexes in an infertile human male carrier of a pericentric inversion inv (1) (p32q42). Hum Genet 76, 81-89

Boer P de, Branje HEB (1979) Association of the extra chromosome of tertiary trisomic male mice with the sex chromosomes during first meiotic prophase, and its significance for impairment of spermatogenesis. Chromosoma (Berl) 73, 369-379

Bourrouillou G, Mansat A, Calvas P, Pontonnier $F$, Colombies $P$ (1987) Anomalies chromosomiques et infertilité masculine. Etude de 1444 sujets. Bull Assoc Anat 71, 29-31

Burgoyne PS, Baker TG (1984) Meiotic pairing and gametogenic failure. In: Controlling Events in Meiosis. Proc 38th Symp, Reading, Compag of Biologists (Evans CW, Dickinson HG, eds) Cambridge, 349-362

Burgoyne PS, Mahadevaiah S, Mittwoch UA (1985) Reciprocal autosomal translocation 
which causes male sterility in the mouse also impairs oogenesis. $J$ Reprod Fertil 75,647 . 665

Chandley AC (1979) The chromosomal basis of human infertility. Br Med Bull 35, 181-186

Chandley AC, Speed RM, McBeath S, Hargreave TB (1986) A human 9; 20 reciprocal translocation associated with male infertility analyzed at prophase and metaphase 1 of meiosis. Cytogenet Cell Genet 41, 145-153

Chandley AC, McBeath S, Speed RM, Yorston L, Hargreave TB (1987) Pericentric inversion in human chromosome 1 and the risk for male sterility. J Med Genet 24, 325-334

Davisson MT, Poorman PA, Roderick TH, Moses MJ (1981) A pericentric inversion in the mouse. Cytogenet Cell Genet 30, 70-76

Devictor-Vuillet M, Luciani JM, Carlon N, Stahl A (1971) Anomalies de structure et rôle du chromosome $Y$ chez l'homme. Pathol Biol $19,231-249$

Dutrillaux B (1985) Incidence des anomalies chromosomiques chez les hommes stériles. Contracept Fertil Sex 13, 803-814

Ferguson-Smith MA, Lennox $B$, Mack WS, Stewart JSS (1957) Klinefelter's syndrome. Frequency and testicular morphology in relation to nuclear sex. Lancet ii, 167-169

Forejt J (1974) Non-random association between a specific autosome and the $X$ chromosome in meiosis of the male mouse: possible consequence of the homologous centromeres' separation. Cytogenet Cell Genet 13, 369-383

Forejt J (1979) Meiotic studies of translocations causing male sterility in the mouse II. Double heterozygotes for robertsonian translocations. Cytogenet Cell Genet 23, 163-170

Forejt $J(1982) X-Y$ involvement in male sterility caused by autosome translocations: a hypothesis. In: Serono Clinical Colloquia on Reproduction. III. Genetic Control of Gamete Production and Function (Crosignani PG, Rubin BL, eds) Academic Press, Grune and Straton, London, 135-151

Forejt J, Gregorova S (1977) Meiotic studies of translocations causing male sterility in the mouse. I. Autosomal reciprocal translocations. Cytogenet Cell Genet 19, 159-179

Forejt J, Gregorova S, Goetz P (1981) XY pair associates with the synaptonemal complex of autosomal male-sterile translocations in pachytene spermatocytes of the mouse (Mus musculus). Chromosoma (Berl) 82, 41-53
Gabriel-Robez O, Ratomponirina C, Rumpler $Y$, LeMarec B, Luciani JM, Guichaoua MR (1986a) Synapsis and synaptic adjustment in an infertile human male heterozygous for a pericentric inversion in chromosome 1. Hum Genet 72, 148-152

Gabriel-Robez O, Ratomponirina C, Dutrillaux B, Carré-Pigeon F, Rumpler Y (1986b) Meiotic association between the $X Y$ chromosomes and the autosomal quadrivalent of a reciprocal translocation in two infertile men, $46, X Y$, $t(19 ; 22)$ and $46, X Y, t(17 ; 21)$. Cytogenet Cell Genet 43, 154-160

Gonzalez J, Lesourd S, Dutrillaux B (1981) Mitotic and meiotic analysis of a reciprocal translocation, $\mathrm{t}(\mathrm{Y}: 3)$, in an azoospermic male. Hum Genet 57, 111-114

Groupe de Cytogénéticiens français (1986) Pericentric inversions in man. A French collaborative study. Ann Genet 29, 129-168

Guichaoua MR, Delafontaine D, Taurelle R, Taillemite JL, Morazzani MR, Luciani JM (1986) Loop formation and synaptic adjustment in a human male heterozygous for two pericentric inversions. Chromosoma (Berl) 93, 313-320

Hartung M, Devictor M, Codaccioni JL, Stahl A (1988) $\mathrm{Yq}$ deletion and failure of spermatogenesis. Ann Genet 31, 21-26

Hook EB, Hamerton JL (1977) The frequency of chromosome abnormalities detected in consecutive newborn studies. Differences between studies. Results by sex and severity of phenotypic involvements. In: Population $\mathrm{Cy}$ togenetics: Studies in Humans (Hook EB, Porter IH, eds) Academic Press, New York, San Francisco, London, 63-79

Hotta Y, Chandley AC (1982) Activities of Xlinked enzymes in spermatocytes of mice rendered sterile by chromosomal alterations. Gamete Res 6, 65-72

Jacobs PA, Strong JA (1959) A case of human intersexuality having a possible $X X Y$ sexdetermining mechanism. London. Nature 183, 302-303

Johannisson R, Gropp A, Winking $H$, Coerdt W, Rehder H, Schwinger E (1983) Down's syndrome in the male. Reproductive pathology and meiotic studies. Hum Genet 63, 132-138

Johannisson R, Löhrs $U$, Wolff $H H$, Schwinger $E$ (1987) Two different $X Y$-quadrivalent associations and impairment of fertility in men. $C y$ togenet Cell Genet 45, 222-230

Johannisson R, Löhrs U, Passarge E (1988) Pachytene analysis in males heterozygous 
for a familial translocation $(9 ; 12 ; 13)$ (q22;q22;q32) ascertained through a child with partial trisomy 9. Cytogenet Cell Genet 47, 160-166

Kjessler B (1972) Facteurs génétiques dans la subfertilité mâle humaine. In: Fécondité et Stérilité du Mâle. Acquisitions Récentes. Masson, Paris, 205-225

Koulischer L, Schoysman R (1975) Etude des chromosomes mitotiques et méiotiques chez les hommes infertiles. $J$ Genet Hum 23 suppl, $58-70$

Laurent C, Chandley AC, Dutrillaux B, Speed RM (1982) The use of surface spreading in the pachytene analysis of a human, $t(Y: 17)$ reciprocal translocation. Cytogenet Cell Genot 33, 312-318

Lifschytz E, Lindsley DL (1972) The role of Xchromosome inactivation during spermatogenesis. Proc Natl Acad Sci USA 69, 182186

Luciani JM, Guichaoua MR, Mattei A, Morazzani MR (1984) Pachytene analysis of a man with a 13q,14q translocation and infertility. Behavior of the trivalent and non-random association with the sex vesicle. Cytogenet Cell Genet $38,14-22$

Luciani JM, Guichaoua MR, Delafontaine D, North MO, Gabriel-Robez O, Rumpler $Y$ (1987) Pachytene analysis in a 17;21 reciprocal translocation carrier: role of the acrocentric chromosomes in male sterility. Hum Genet 77, 246-250

Marmor D, Taillemite JL, Van Den Akker J, Portnoi MF, Le Porrier N, Joye N, Delafontaine D, Roux C (1980) Semen analysis in subfertile balanced translocation carriers. Fertil Steril 34, 496-502

Miklos GLG (1974) Sex-chromosome pairing and male fertility. Cytogenet Cell Genet 13, 558-577

Mittwoch U, Mahadevaiah S, Setterfield LA (1984) Chromosomal anomalies that cause male sterility in the mouse also reduce ovary size. Genet Res 44, 219-224

Quack B, Speed RM, Luciani JM, Noel B, Guichaoua MR, Chandley AC (1988) Meiotic analysis of two human reciprocal $X$ autosome translocations. Cytogenet Cell Genet 48, 43-47

Ratomponirina C, Couturier J, Gabriel-Robez $\mathrm{O}$, Rumpler $\mathrm{Y}$, Dutrillaux $\mathrm{B}$, Croquette $\mathrm{M}$, Rabach Q, Leduc M (1985) Aberrations of the synaptonemal complexes in a male 46, $\mathrm{XY}, \mathrm{t}(\mathrm{Y:14})$. Hum Genet 2, 214-218

Retief AE, Van Zyl JA, Menkveld R, Fox MF, Kotze GM, Brusnicky J (1984) Chromosome studies in 496 infertile males with a sperm count below $10 \mathrm{million} / \mathrm{ml}$. Hum Genet 66, 162-164

Rosenmann A, Wahrman J, Richler C, Voss R, Persitz A, Goldman B (1985) Meiotic association between the XY chromosomes and unpaired autosomal elements as a cause of human male sterility. Cytogenet Cell Genet 39, 19-29

Saadallah N, Hulten M (1985) A complex three breakpoints translocation involving chromosomes 2,4 and 9 identified by meiotic investigations of a human male ascertained for subfertility. Hum Genet 71, 312-320

Soler A, Salami C, Balmes I (1981) Pericentric X chromosome in a family. Clin Genet 20, 234239

Speed RM (1988) The possible role of meiotic pairing anomalies in the atresia of human fetal oocytes. Hum Genet 78, 260-266

Tiepolo L, Zuffardi O (1976) Localization of factors controlling spermatogenesis in the nonfluorescent portion of the human $Y$ chromosome long arm. Hum Genet 34, 119-124

Viguié F, Romani F, Dadoune JR (1982) Male fertility in a case of $(Y ; 6)$ balanced reciprocal translocation. Mitotic and meiotic study. Hum Genet 62, 225-227

Zuffardi O, Tiepolo L (1982) Frequencies and types of chromosome abnormalities associated with human male infertility. In: Serono Clinical Colloquia on Reproduction. III. Genetic Control of Gamete Production and Function (Crosignani PG, Rubin BL, eds) Academic Press and Grune and Stratton, London, 261-273 